Ann. Biol. anim. Bioch. Biophys., I964, 4 (3) 295-305.

\title{
CHROMATOGRAPHIE DES PROTÉINES DU LACTOSÉRUM DE BREBIS
}

\author{
J. L. MAUBOIS (1) \\ avec la collaboration technique de C. Bourllanne et de Barbara Nistchike \\ Station centrale de Recherches laitieres et de Technologie des Produits animaux, \\ Centre national de Recherches zootechniques, Jouy-en-Josas (Seine-et-Oise)
}

\section{SOMMAIRE}

La séparation des protéines du lactosérum de brebis sur colonne de D.E.A.E.-cellulose a été étudiée. Douze fractions peuvent être décelées. Leur homogénéité a été étudiée par électrophorèse sur papier. Cette dernière technique, complétée par la spectrophotométrie en UV et la recherche des anticorps agglutinants et antitoxiques, a permis l'identification des pics correspondant à la lactoperoxydase, aux immuno-globulines, à la $\beta$-lactoglobuline et à la sérum-albumine. La séparation de constituants à base d'uridine et de guanine, non dialysables, a été obtenue par filtration sur Sephadex G roo.

\section{INTRODUCTION}

Peu d'études analytiques ont été consacrées aux protéines du lait de brebis. Deutsch (I947) par électrophorèse selon TISELIUS décèle huit constituants dont il donne les mobilités. Diranyan et Agababyan (I962) séparent et dosent 4 fractions seulement par électrophorèse sur papier. RAvaroli et GIOvENALI (I96I) observent des différences de mobilités électrophorétiques entre les protéines du lactosérum de vache et celles du lactosérum de brebis et notent chez la brebis, l'importance relative d'un constituant qui, à lui seul, représente $73 \mathrm{p}$. Ioo des protéines du lactosérum.

Notre travail avait pour but de mettre au point une technique de fractionnement et de dosage des immuno-globulines qui soit à même de confirmer les résultats obtenus par PLOMMET (I964) sur la synthèse des anticorps dans la mamelle. Nous avons été conduits à étudier les protéines du lactosérum de brebis à l'aide d'une méthode de séparation différente de l'électrophorèse (sur papier ou selon TISELIUS), la chromatographie sur échangeurs d'ions.

(1) Adresse actuelle : Laboratoire de Recherches de Technologie Laitière, Centre de Recherches agronomiques de l'Ouest, Rennes, France. 
L'homogénéité des pics isolés par chromatographie sur colonne de D.E.A.E. cellulose a été étudiée par électrophorèse sur papier. Les meilleures séparations sur colonne ayant été obtenues à un $\mathrm{pH}$ voisin de celui de l'électrophorèse, l'interprétation des diagrammes obtenus avec les deux techniques s'en est trouvée facilitée.

\section{MATÉRIEL ETT TECHNIQUES}

\section{Préparation des protéines du lactosérum}

Deux types de préparation ont été réalisés à partir de laits de mélange ou individuels de brebis appartenant au troupeau du C. N. R. Z. (race Préalpes du Sud).

a) Précipitation selon Aschaffenburg et DREWry (1959). - Le volume préconisé par les auteurs pour le lait de vache a été modifié. A $25 \mathrm{ml}$ de lait entier, dilués à $80 \mathrm{ml}$ et portés à $35^{\circ} \mathrm{C}$, on ajoute en agitant $4 \mathrm{ml} \mathrm{de} \mathrm{CH}_{3} \mathrm{COOH}$ a Io p. Ioo, puis 5-Io minutes plus tard, $4 \mathrm{ml} \mathrm{de} \mathrm{CH}_{3} \mathrm{COONa}$; on complète à $100 \mathrm{ml}$. Après refroidissement, on filtre sur papier Whatman $\mathrm{n}^{\circ} 3 \mathrm{MM}$.

b) Précipitation de la caséine avec $\mathrm{HCl} \mathrm{N}$. - Le lait écrémé, dilué au $\mathrm{I} / 2$, chauffé à $35^{\circ} \mathrm{C}$ est amené à $\mathrm{pH} 4,5$ avec $\mathrm{HCl} \mathrm{N}$. Ie surnageant de centrifugation est filtré sur papier Whatman $n^{0} 3 \mathrm{MM}$.

Les 2 types de filtrats sont dialysés d'abord contre l'eau courante (I nuit), puis contre l'eau distillée pendant 4-5 jours ( 5 fois 20 volumes). Le dialysat est de nouveau filtré sur papier. In effet, un précipité très ninime, dont la nature n'a pas été étudiée, se forme au cours de la dialyse. Le filtrat est lyophilisé. Les produits lyophilisés sont conservés au réfrigérateur $\left(+4^{\circ} \mathrm{C}\right)$.

\section{Chromatographie sur colonne de D.E.A.E.-cellulose}

La D.E.A.E.-cellulose SERva est lavée par la soude et par le tampon pipérazine- $\mathrm{NaCl} \mathrm{pH} 4,4$ selon Seymnza (ig60). Des colonnes de $3 \times 20 \mathrm{~cm}$ ont été utilisées. Les chromatographies ont été réalisées à la température du laboratoire soit en tampon Tris o,o I $\mathrm{M}$ - $\mathrm{HCI} \mathrm{pH} 8,5$ soit en tampon pipérazine 0,0067 M-HCI pH 8,5. Aucune influence propre au tampon, sur la séparation n'a été constatée.

600 à $800 \mathrm{mg}$ de produit lyophilisé, dissous dans ıo $\mathrm{ml}$ de tampon sont placés sur la colonne. Une élution par gradient exponentiel de force ionique $(\mathrm{NaCl} o \rightarrow 0,4 \mathrm{M})$ a été employée. La vitesse d'élution était de $60 \mathrm{ml} / \mathrm{h}$ et des fractions de $10 \mathrm{ml}$ étaient collectées. Ie volume du mélangeur était de $2000 \mathrm{ml}$.

Dans la plupart des chromatographies, les deux fractions de fin d'élution, qui, avec ce gradient, sortent très étalées ont été rassemblées en quelques tubes, en éluant directement avec le tampon $0,4 \mathrm{H} \mathrm{NaCl}, 20$ à 30 tubes après la sortie du pic F8 (fig. I et 2).

\section{Filtration sur Sephadex $G$ Ioo}

Une colonne de $3 \times 5^{6} \mathrm{~cm}$ est remplie avec du Sephadex G roo que l'on a laissé gonfler dans le tampon pipérazine $0,0067 \mathrm{M}$ - $\mathrm{HCl}$ pH $8,5 \mathrm{NaCl} 2 \mathrm{M}$ durant deux jours. $300 \mathrm{mg}$ de produit dissous dans $3 \mathrm{ml}$ du même tampon sont placés sur la colonne.

\section{Électrophorèse sur papier}

Les expériences ont été réalisées en tampon Véronal-Véronal sodique pH 8,6 selon AschaFFENBURG et DREWRY (1955).

\section{Recherche de l'activité peroxydasique}

Méthode au gaïacol (Maehly et Chance - I954). 
Recherche des anticorps

Le lait utilisé venait de brebis vaccinées par plusieurs injections locales ou générales d'antigènes salmonelliques et brucelliques et d'anatoxine staphylococcique. La détection et le dosage des anticorps correspondants (Salmonella $\mathrm{O}$ et $\mathrm{H}$, Brucella) ont été faits par agglutination et par inhibition de l'hémolyse due à la toxine $\alpha$ (PLOMMET 1964). Les titrages ont été réalisés à partir de $5 \mathrm{mg}$ de produit lyophilisé dissous dans $0,85 \mathrm{ml}$ d'eau.

\section{Détermination des coefficients d'extinction}

Les fractions lyophilisées ont été au préalable desséchées $48 \mathrm{~h}$ sous vide et $\mathrm{P}_{2} \mathrm{O}_{5}$ puis dissoutes dans un tampon Tris $0,0 \mathrm{I} \mathrm{M}-\mathrm{HCl} \mathrm{pH} 8,5$ pour les Io premiers pics, dans la soude $\mathrm{N} / 4$ pour les deux derniers. Les lectures ont été faites à l'aide du spectrophotomètre Cary $\mathbf{4} 4$.

\section{RÉSULTATS}

43 chromatographies de lactosérum provenant les unes de laits de mélange, les autres de 22 laits individuels ont été réalisées.

Les chromatogrammes les plus représentatifs des séparations obtenues avec les deux types de lactosérum sont présentés dans les figures I et 2.95 à Ioo p. Ioo en poids des protéines placées sur la colonne sont éluées et I2 pics peuvent être dénombrés. Les tubes correspondant aux sommets des pics ont été rassemblés, dialysés et lyophilisés. Les fractions ainsi obtenues, ont été étudiées par spectrophotométrie en UV (tabl. I) et par électrophorèse sur papier (fig. 3). Nous avons également recherché l'activité peroxydasique et la présence d'anticorps agglutinants et antitoxiques.

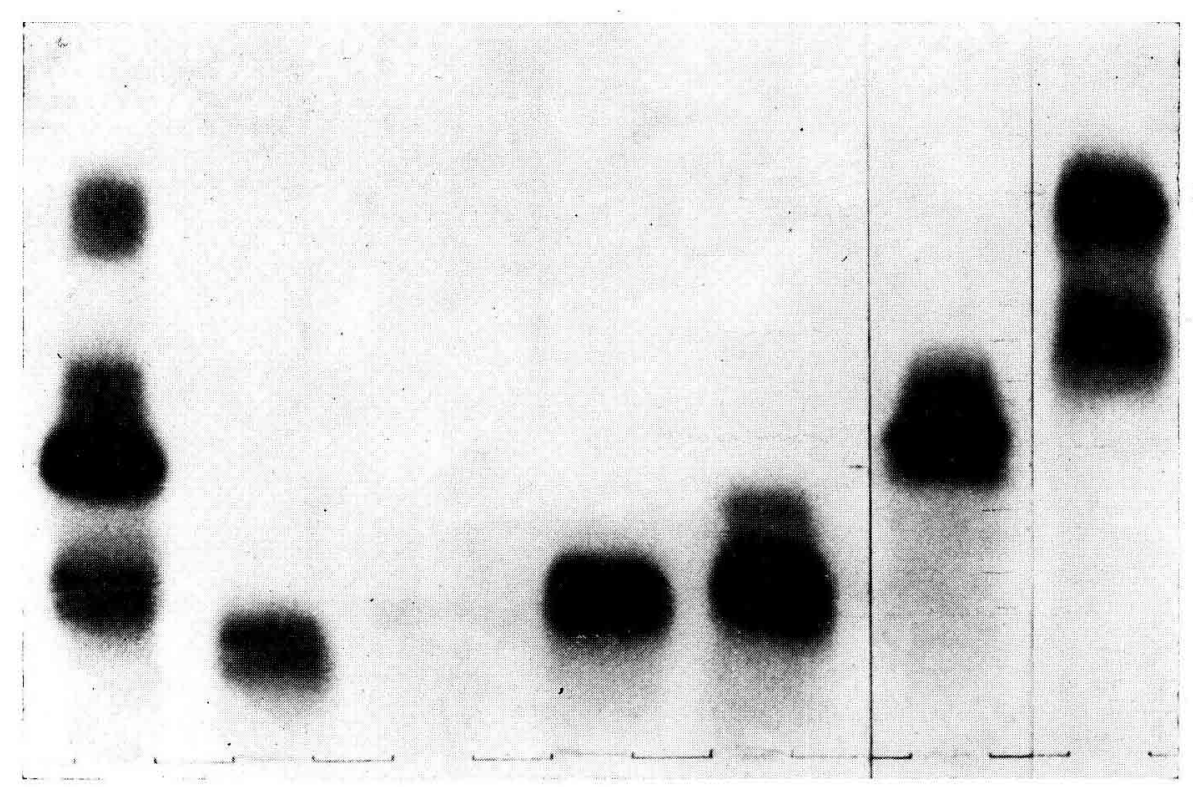

FIG. 3. - Electrophorèse sur papier de diftérentes fractions isolées par chromatographie sur colonne De gauche à droite : lactosérum total L. T., (filtrat $\mathrm{pH}_{4,5}$ )

pic $F_{1}-$ pic $F_{2}-$ pic $F_{4}-$ pics $F_{5-6-7}$ réunis - pic $\mathrm{F}_{8}-$ pic $\mathrm{F}_{9-10}$ réunis.

$\mathrm{F}_{2}$ constitué principalement de nucléotides ne se colore pas au bleu de bromophénol. 


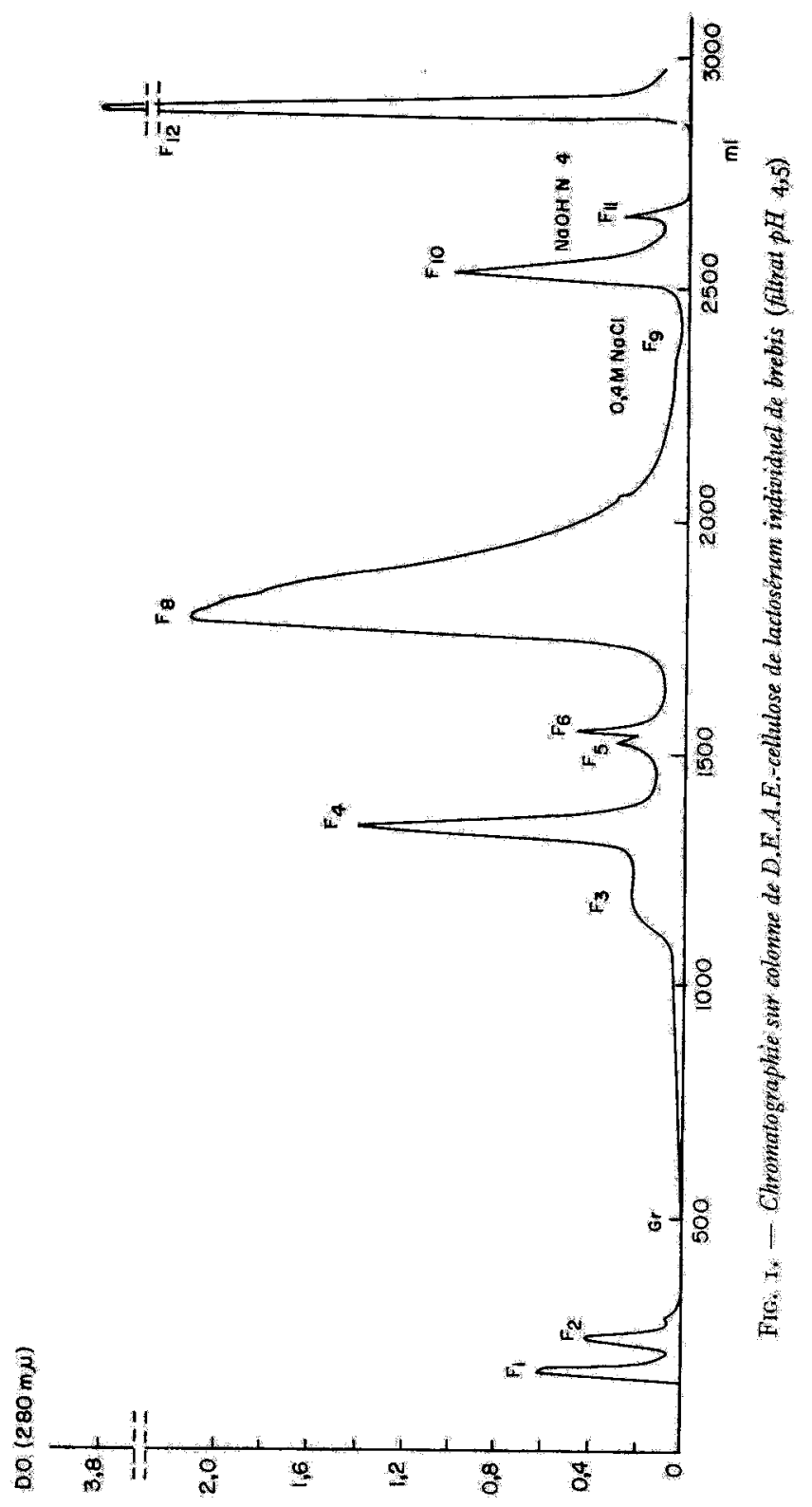


TABIEAU I

Coefficients d'extinction à $280 \mathrm{~m} \mu$ et au maximum d'absorption des fractions isolées par chromatographie sur D.E.A.E.-cellulose

\begin{tabular}{|c|c|c|c|c|c|c|c|c|c|}
\hline Pics & $F_{1}$ & $\mathrm{~F}_{2}$ & $F_{3}$ & $\mathrm{I}:$ & $F_{5-6-7}$ & $\mathrm{~F}_{8}$ & $F_{9-10}$ & $F_{11}$ & $F_{12}$ \\
\hline $\begin{array}{c}\operatorname{Max}(\mathrm{m} \mu) \\
\text { E } 0,1 \% 1 \mathrm{~cm}, 280 \mathrm{~m} \mu\end{array}$ & $\begin{array}{l}278 \\
0,93\end{array}$ & $\begin{array}{l}262 \\
1,40\end{array}$ & $\begin{array}{l}270 \\
0,60\end{array}$ & 2638 & $\begin{array}{l}278 \\
0,89\end{array}$ & $\begin{array}{l}278 \\
0,81\end{array}$ & $\begin{array}{c}278 \\
0,75\end{array}$ & $\begin{array}{c}290 \\
0,97\end{array}$ & $\begin{array}{l}290 \\
1,07\end{array}$ \\
\hline E $0,1 \% 1 \mathrm{~cm}, \lambda \max$ & 0,98 & 3,17 & 0,67 & 2,72 & 0,90 & 0,81 & 0,79 & 1,05 & $1,1^{\prime}$ \\
\hline
\end{tabular}

\section{$0.0 .(280 \mathrm{m \mu})$}

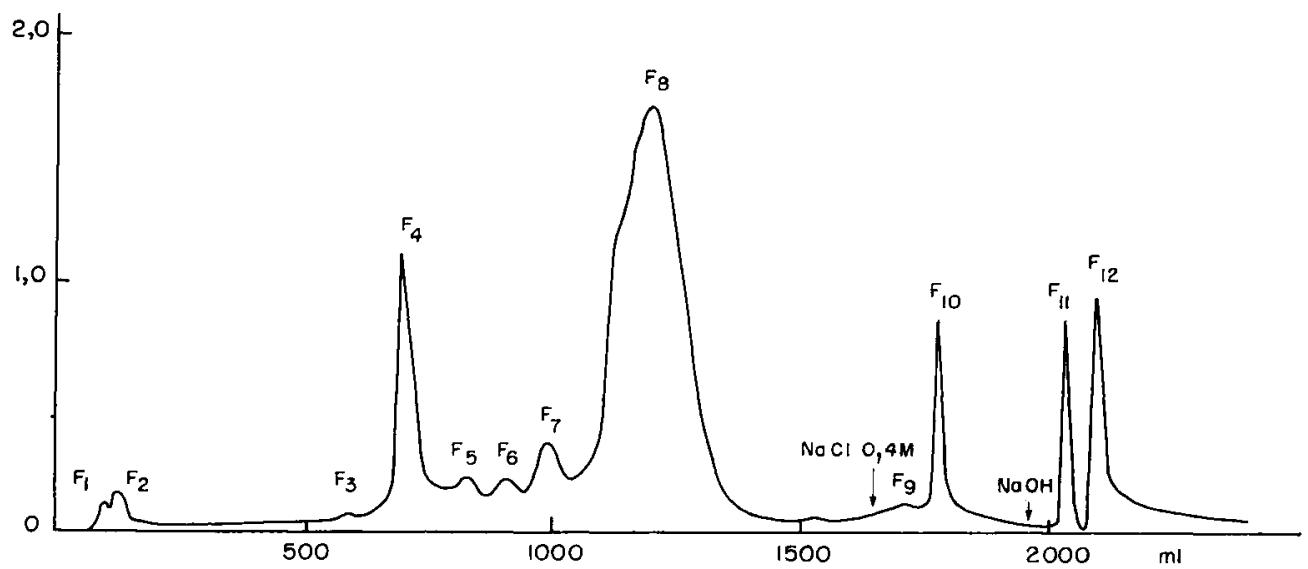

FIG. 2. - Chromalographie sur colonne de D.E.A.E.-cellulose de lactosérum de mélange (précipitation selon Aschaffenburg et al.)

Le pic $F_{1}$ présente une activité peroxydasique. Si la peroxydase du lait de brebis a la même activité spécifique que celle du lait de vache, cette protéine représenterait Io $\mathrm{p}$. Ioo de la fraction $\mathrm{F}_{1}$. L'électrophorèse sur papier montre que cette fraction comporte au moins deux constituants.

Les pics $\mathrm{F}_{2}-\mathrm{F}_{3}-\mathrm{F}_{4}$ présentent un spectre d'absorption à caractère nucléique. Pour vérifier la présence possible de nucléotides dans nos préparations de lactosérum, une filtration sur Séphadex G Ioo à force ionique élevée a été réalisée, ceci dans le but de dissocier, s'il y avait lieu, les nucléoprotéines présentes. Le diagramme obtenu est donné par la figure 4. La fraction ayant un maximum d'absorption à $260 \mathrm{~m} \mu$, représentant $9,5 \mathrm{p}$. Ioo de la somme des densités optiques à $280 \mathrm{~m} \mu$, a été dialysée et lyophilisée. La guanine et l'uracile monophosphate ont pu être identifiés après hydrolyse par 1'acide chlorhydrique $\mathrm{N}$ I h à $100^{\circ}$ et chromatographie en isopropanol/ $\mathrm{HCl} / \mathrm{H}_{2} \mathrm{O}$. Le rapport $\mathrm{U} / \mathrm{G}$ est de l'ordre de 2 . 


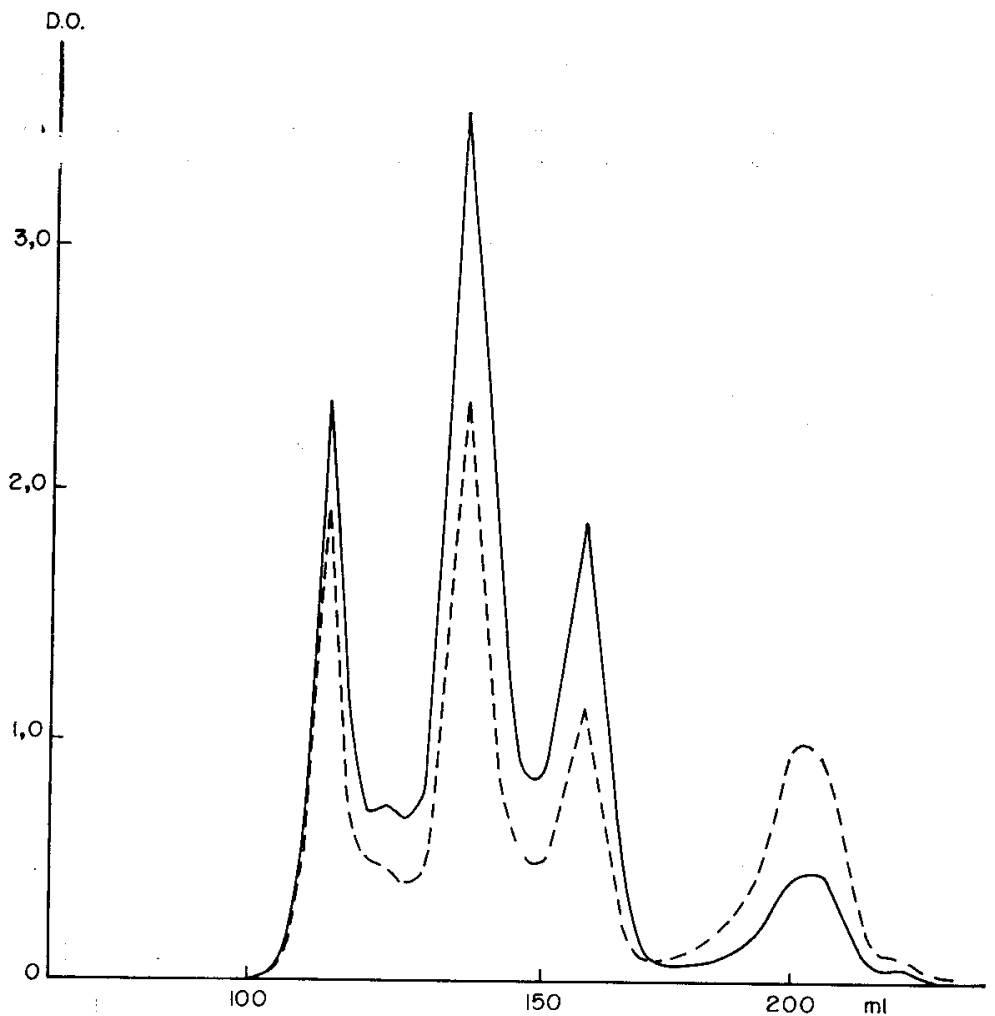

FIG. 4. - Filtration sur Séphadex $G$ Ioo de lactosérum de brebis (fultrat pH 4,5) Fractions de ro $\mathrm{ml}-$ Vitesse de filtration $40 \mathrm{ml} /$ heure.

Densité optique à $280 \mathrm{~m} \mu$.

Densité optique à $260 \mathrm{~m} \mu$.

\section{TABLEAU 3}

Agglutination et inhibition de l'hémolyse due à la toxine $\alpha$ présentées par les différenles fractions isolées par chromatographie sur colonne.

\begin{tabular}{|c|c|c|c|c|}
\hline \multirow{2}{*}{ Fractions } & \multicolumn{3}{|c|}{ Dilution d'agglutination avec : } & \multirow{2}{*}{$\begin{array}{l}\text { Dilution n'inhibant } \\
\text { plus l'hémolyse due } \\
\text { à la toxine } \alpha\end{array}$} \\
\hline & Antigsene 0 & Antigrine HI & $\begin{array}{c}\text { Antigène } \\
\text { Brucellique }\end{array}$ & \\
\hline 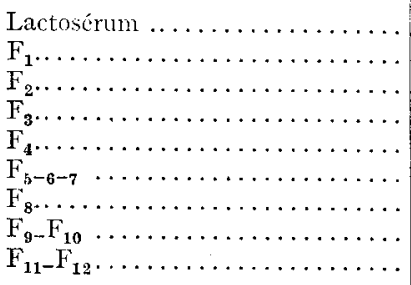 & $\begin{array}{c}1 / 8 \\
0 \\
0 \\
1 / 4 \\
0 \\
1 / 4 \\
0 \\
0 \\
0\end{array}$ & $\begin{array}{c}1 / 8 \\
0 \\
0 \\
1 / 256 \\
1 / 256 \\
1 / 32 \\
0 \\
1 / 4 \\
0\end{array}$ & $\begin{array}{c}1 / 16 \\
0 \\
0 \\
1 / 128 \\
1 / 64 \\
1 / 64 \\
0 \\
0 \\
0\end{array}$ & $\begin{array}{c}1 / 16 \\
1 / 2 \\
0 \\
1 / 32 \\
1 / 32 \\
1 / 8 \\
- \\
- \\
-\end{array}$ \\
\hline
\end{tabular}


Les pics $\mathrm{F}_{3}$ et $\mathrm{F}_{4}$ contiennent la majorité des anticorps agglutinants et antitoxiques. Une légère agglutination correspondant probablement à des traces de ces fractions est observée avec les pics $\mathrm{F}_{5}-\mathrm{F}_{6}$ et $\mathrm{F}_{7}$ (tabl. 3)

Les pics $\mathrm{F}_{5}-\mathrm{F}_{6}-\mathrm{F}_{7}$ ne sont présents que dans le lactosérum provenant de laits de mélange. Dans le cas des lactosérum individuels étudiés, $\mathrm{I}$, voire 2 de ces pics sont souvent absents. I'électrophorèse sur papier de ces 3 pics réunis ne présente que 2 bandes d'inégale importance.

\section{TABLEAU 2}

\section{Proportion des différents pics isolés par chromatographie dans} les deux types de lactosérum

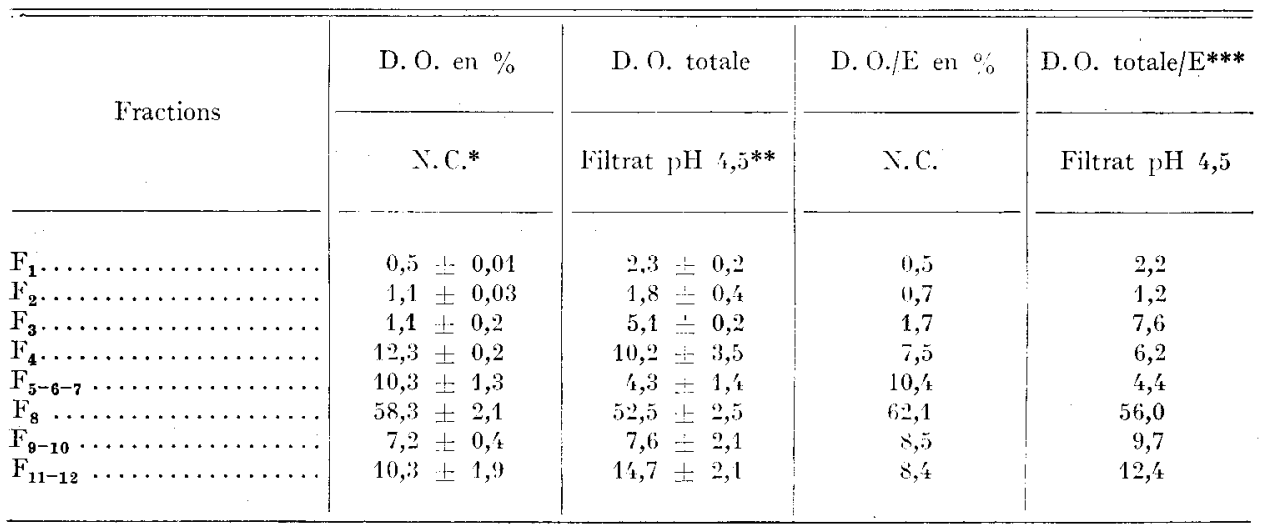
(1959).

* N.C. = (Non caséine) lactoscrum résultant de la précipitation selon Ascinafiexblra et Drewry

** Filtrat $\mathrm{pH} 4,5$ : précipitation isoélectrique à $\mathrm{pH}^{4}, 5$ jar IICl $\mathrm{x}$.

*** Coefficient d'extinction à 280 mụ.

Le pic $\mathrm{F}_{8}$ représente le constituant principal du lactosérum (environ $60 \mathrm{p}$. roo). I1 présente un épaulement en chromatographie et 3 bandes sont décelées par électrophorèse sur papier. Une préparation de $\beta$-lactoglobuline de brebis cristallisée (MAUBoIs et al., I964) migre au niveau de la bande centrale.

Les pics $\mathrm{F}_{9}$ et $\mathrm{F}_{10}$ sont difficilement séparables avec le gradient utilisé, aussi les avons nons étudiés ensemble. Deux bandes sont décelées en électrophorèse sur papier. L'examen d'un sérum sanguin avec cette même technique nous a permis đe constater d'une part que le composant le plus rapide avait la même mobilité que la sérum albumine et d'autre part que les pics $F_{3}$ et $F_{4}$ qui ont tous les deux une mobilité électrophorétique identique migraient au niveau des bandes des immuno-globulines du sérum.

La proportion des différents pics séparés par chromatographie sur colonne dans les deux types de lactosérum est donnée dans le tableau 2 . Les résultats énoncés sont la moyenne de 4 expériences réalisées à partir de la même préparation de lactosérum provenant de lait de mélange. Lors de l'examen des lactosérum individuels des variations quantitatives très importantes ont été constatées au niveau des pics $\mathrm{F}_{2}$ et $\mathrm{F}_{4}$. 
Les valeurs extrêmes sont de l'ordre de 0,7 à $7 \mathrm{p}$. Ioo (DO/E) pour $\mathrm{F}_{2}$, de 2,4 à 6,9 p. Ioo pour $\mathrm{F}_{4}$.

Io à I4 p. Ioo du produit mis sur la colonne ne sont élués que par la soude $\mathrm{N} / 4$. Cette proportion atteint $17 \mathrm{p}$. roo pour certains lactosérum individuels.

\section{DISCUSSION}

La chromatographie sur colonne permet de séparer le lactosérum de brebis en au moins Io fractions dont deux sont des constituants à base d'uracile et de guanine. Six des pies peuvent être identifiés au moyen de l'électrophorèse sur papier, de la spectrophotométrie en $\mathrm{CV}^{\prime}$ et par la recherche des anticorps.

DENAMUR et al., (I959) ont rapporté la présence en quantité relativement importante de nucléotides à base de guanine et d'uracile dans le lait de brebis $(27,2$ micromoles de $\mathrm{G}$ et 262 micromoles de $\mathrm{U}$ pour Ioo $\mathrm{ml}$ de lait). Environ $25 \mathrm{p}$. Ioo des nucléotides guanyliques et $5 \mathrm{p}$. Ioo des nucléotides uridyliques décelés par ces auteurs sont encore présents dans nos préparations. Il parait exclu que ces composés soient liés directement à des protéines; en effet, une filtration sur Séphadex G Ioo dans le même tampon pipérazine mais à force ionique faible $(\mu=0,0 I)$ a donné une séparation identique à celle de la figure 4 . Il est plus probable que ces nucléotides sont liés à des sucres ou des dérivés sucrés pour former des composés du type guanosine hexose diphosphate, uridine diphosphate sucre et uridine diphosphate acétyl hexosamine comme ceux isolés par DENAMur et al., (I959). Ces constituants seraient adsorbés sur les molécules protéiques et de ce fait non dialysables.

L'élimination de ces composés du lactosérum de brebis se fait facilement par passage sur Séphadex G Ioo et il est probable qu'une séparation plus fine des protéines serait obtenue en chromatographiant chacun des 3 pics obtenus par ce dernier traitement.

L'examen de l'absorption en UV montre que ces nucléotides sortent au niveau des pics $\mathrm{F}_{2}-\mathrm{F}_{3}$ et $\mathrm{F}_{4}$. I es pics $\mathrm{F}_{2}$ et $\mathrm{F}_{4}$ doivent leur être attribués en majeure partie.

Le pic $\mathrm{F}_{1}$ est un mélange de deux ou plusieurs protéines dont la lactoperoxydase. La chromatographie sur colonne de D.E.A.E.-cellulose pourrait être un premier stade de purification dans la préparation de cet enzyme qui n'a pas encore été isolé du lait de brebis.

La présence d'anticorps et la comparaison par électrophorèse sur papier avec un sérum sanguin de brebis indiquent que les immuno-globulines sont localisées au. niveau des pics $\mathrm{F}_{3}$ et $\mathrm{F}_{4}$.

Le pic $F_{8}$ qui est formé essentiellement par la $\beta$-lactoglobuline, présente trois bandes par électrophorèse sur papier. Or deux bandes sont décelées dans l'électrophorèse sur papier et sur gel d'acrylamide de $\beta$-lactoglobuline cristallisée, préparée à partir d'un lait de petit mélange (Maubois et $a l$., I 964 ). Seul l'examen de nombreux laits individuels pourrait nous permettre d'affirmer que ces deux bandes correspondent à des variants génétiques de même nature que ceux identifiés dans le lait de vache (ASchafFenbuRG et DREWRY, I957). Cependant l'épaulement constaté en chromatographie et deux des 3 bandes décelées en électrophorèse sur papier de la. 
fraction $\mathrm{F}_{\mathbf{8}}$ doivent pouvoir être attribués en toute vraisemblance à ce type d'hétérogénéité. Quant à la $3^{\mathrm{e}}$ bande décelée en électrophorèse sur papier, il est probable qu'il s'agit d'une impureté de nature différente de la $\beta$-lactoglobuline. La proportion de 56 à $60 \mathrm{p}$. Ioo calculée pour ce pic $\mathrm{F}_{8}$ semble plus valable que celle déterminée par Ravaior I et Giovenali (I96I) à partir d'électrophorèses sur papier. Ces auteurs n'ont en effet, pas tenu compte des divers coefficients de fixation du colorant par les différentes protéines du lactosérum.

Le pic $F_{10}$ sortant le dernier au cours de l'élution doit correspondre au composant le plus rapide en électrophorèse sur papier, et de ce fait, représenter la sérumalbumine contenue dans le lait de brebis. Les pics $F_{5}-F_{6}-F_{7}$ et $F_{9}$ n'ont pu être identifiés. Quant au matériel élué par la soude $\left(\mathrm{F}_{11}, \mathrm{~F}_{12}\right)$, il peut s'agir soit de protéines particulières contenues dans le lactosérum, soit d'une fraction représentative du produit de départ fortement adsorbée sur le support et ne pouvant être éluée par l'élévation de force ionique.

La différence très reproductible de comportement sur colonne de D.E.A.E.cellulose entre les 2 types de lactosérum s'explique difficilement. Cette différence est supérieure aux erreurs d'expérience (cf tabl. 2 oì les écarts maximums observés sur les essais sont donnés pour chaque pic). Dans la précipitation selon AschafFENBURG et DREWRY (I959), une floculation brutale de la caséine à $\mathrm{pH}$ 4, I a lieu et i1 se peut que certaines protéines correspondant aux pics $F_{1}-F_{2}-F_{3}$ soient entraînées partiellement par adsorption lors de ce traitement, mais cette hypothèse n'est guère satisfaisante car la fraction $\mathbf{F}_{5}-\mathrm{F}_{6}-\mathrm{F}_{7}$ présente une proportion plus élevée dans ce type de lactosérum.

La chromatographie sur colonne de D.E.A.E.-cellulose semble donc être un moyen commode et simple de séparer les protéines du lactosérum de brebis ; un meilleur fractionnement, obtenu après élimination des constituants nucléotidiques à base d'uridine et de guanine doit nous permettre de caractériser les pics non encore identifiés. La séparation et la purification des différentes protéines du lactosérum et plus particulièrement des immuno-globulines peuvent être espérées à partir des résultats déjà obtenus avec cette technique.

Reçu pour publication en mars 19604.

\section{REMERCIEMENTS}

Nous tenons à exprimer nos remerciements à M. GAYE qui a bien voulu se charger de l'identification des nucléotides isolés par filtration sur Séphadex et à Mme VASSAL pour la recherche de la lactoperoxydase.

Nous tenons également à remercier MM. Plommet, Ribadeau-Dumas et Garnier pour l'aide et les conseils qu'ils nous ont apportés dans la réalisation de ce travail.

\section{SUMMARY}

CIIROMATOGRAPHY OF WHEY PROTEINS OF EWE'S MILK

The separation of whey proteins of ewe's milk on D.E.A.E.-cellulose column has been studied. Twelve tractions have been detected. Their purity has been studied by paper electrophoresis. The fractions corresponding to Lactoperoxydase, Immune globulins, $\beta$-Lactoglobulin and Serum albumin 
have been identified by paper electrophoresis, U. V. Spectrophotometry and precipitins and antitoxins antibodies tests. The fractions $\mathbf{F}_{\mathbf{2}}, \mathrm{F}_{\mathbf{3}}$ and $\mathrm{F}_{\mathbf{4}}$ contain non dialysable components with Uridine and Guanine, they are separated from the whey proteins by filtration on Sephadex $G$ 100. The percent by weight of each fraction has been calculated and $\beta$-Lactoglobulin (fraction 8) repr sents 55 to $00 \mathrm{p}$. 100 of the total proteins of whey of ewe's milk.

\section{RÉFÉRENCES BIBLIOGRAPHIQUES}

Asciaffenburg; R., Drewry J., I955. Occurence of different $\beta$-lactoglobulins in cow's milk. Nature, I $76-2$ г 8 .

Aschaffenburg R., Drewry J., 1959. New procedure for the routine determination of the various

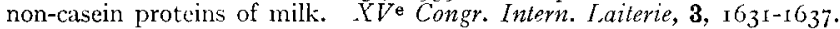

AschnfFendurg R. et DREwry J., 1957. Genetics of the $\beta$-lactoglobulin. Nature, 180-376. 3

Denamur R., kacconseau G. et Guntz G., i959. Les nucléotides acido-solubles des laits de prebis, vache, chèvre et truie. Rev. Esp. Fisiol., 15, 4, 30I-310.

Deutscir H. F., I947. A study of whey proteins from the milk of various animals. J. Biol. Chem., 169, $437-439$.

Dilanyan Z., et Agababrin A., ig62. Investigations on proteins of buffalo's and ewe's milk. $X V^{\text {e }}$ Congr. Intern. Laiterie B, $691-697$.

Maeirly A. C. et Chance B., i954. The Assay of catalases and peroxydases. Melhods Biochem. 'Anal., $1,385-395$.

Maubois J. L., Pion R. et Ribadeau-Dumas B., ig64. En cours de publication.

Plommet M., I964. Lin cours de publication.

Ravaiol L. et Giovenali E., ig6r. Electrophoretic analysis of cow's, ewes' and buffaloes' milk serum. Ist. Sup. San., 24, 748-753.

Semenza G., 1960. Chromatographhie des protéines sur dérivés de la cellulose échangeurs d'ions. Chimia, 14, 325-339. 